\title{
Examples of dynamic chirality in nuclei
}

\author{
M. S. Yavahchova ${ }^{1, *}, D$. Tonev ${ }^{1,2}, N$. Goutev ${ }^{1}, G$. de Angelis ${ }^{2}, R . K$. Bhowmik $^{3}$, R.P. Singh ${ }^{3}, S$. Muralithar $^{3}, N$. Madhavan $^{3}$,

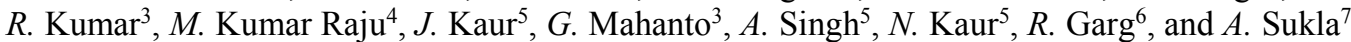 \\ ${ }^{1}$ Institute for Nuclear Research and Nuclear Energy, Bulgarian Academy of Sciences, Sofia, Bulgaria \\ ${ }^{2}$ INFN, Laboratori Nazionali di Legnaro, Legnaro, Italy \\ ${ }^{3}$ Inter-university Accelerator Centre, New Delhi, India \\ ${ }^{4}$ Nuclear Physics Department, Andhra University, Visakhapatnam, India \\ ${ }^{5}$ Department of Physics, Punjab University, Chandigarh, India \\ ${ }^{6}$ Department of Physics\&Astrophysics, Delhi University, New Delhi, India \\ ${ }^{7}$ Department of Physics, Banaras Hindu University, Varanasi, India
}

\begin{abstract}
In many cases the chirality was almost observed but the transition probabilities are different. This fact is clearly seen in the cases of ${ }^{134} \mathrm{Pr}$ and ${ }^{102} \mathrm{Rh}$. In the case of chirality, the yrast and the side bands should be nearly degenerate. In the angular momentum region where chirality sets, the B(E2) values of the electromagnetic transitions deexciting analog states of the chiral twin bands should be almost equal. Correspondingly the B(M1) values should exhibit staggering. Our lifetime measurements in the cases of ${ }^{134} \mathrm{Pr}$ and ${ }^{102} \mathrm{Rh}$ and the theoretical analysis do not support static chirality. Chirality has mainly a dynamical character in both nuclei. In the present paper, we compare our results with the results for other chiral candidate nuclei, which fulfill static chirality conditions.
\end{abstract}

\section{Introduction}

The term "chirality" originates from the Greek word for hand and is a synonym to "handedness". Chirality is recognized as an interesting phenomenon in different branches of science.

Chirality in nuclear physics, has been tested, argued and investigated, both theoretically and experimentally, for more than 20 years.

A spontaneous breaking of chiral symmetry can take place for configurations where the angular momenta of the valence proton, the valence neutron, and the core are mutually perpendicular [1]. Under such conditions, for a triaxial nucleus the short, intermediate and long principal axes form a screw with respect to the angular momentum vector which results in two chiral systems with different orientations, left- and right-handed respectively [2]. Because the chiral symmetry is dichotomic, its spontaneous breaking by the axial angular momentum vector leads to doublets of closely lying rotational bands of the same parity [1-3]. Due to the underlying symmetry, the pairs of chiral twin bands should exhibit systematic properties [4-7].

The first property is the existence of a couple of yrast and side bands, which are nearly degenerate. In the angular momentum region where chirality sets in, the B(E2) values of the electromagnetic transitions deexciting analogue states of the chiral twin bands should be almost equal. Correspondingly the B(M1) values should exhibit odd-even staggering, being much bigger for transitions deexciting states with odd spins than for transitions deexciting states with even spin [6]. The $\mathrm{B}(\mathrm{M} 1)$ values for $\Delta I=1$ transitions connecting the side to the yrast band should have odd-even staggering which is out of phase with respect to the B(M1) staggering for transitions deexciting states in the yrast band and the side band. This is a fingerprint of the ideal static chirality as discussed in $[6,8,9]$

To investigate the presence of chirality in a certain nucleus, it is crucial to determine the $\mathrm{B}(\mathrm{E} 2)$ and $\mathrm{B}(\mathrm{M} 1)$ values.

In many cases, the energy degeneracy of the chiral candidate bands is nearly observed but the corresponding transition probabilities are different, as in the cases of ${ }^{134} \operatorname{Pr}[7,10]$ and ${ }^{102} \mathrm{Rh}[11]$. In the present paper we will investigate the similarity between ${ }^{102} \mathrm{Rh},{ }^{134} \mathrm{Pr}$ and some good candidates to express chirality in nuclei, like ${ }^{135} \mathrm{Nd}$, ${ }^{128} \mathrm{Cs}$ and ${ }^{194} \mathrm{Tl}$.

A good candidate for chiral symmetry nucleus is characterized by the observation of two $\Delta I=1$ bands of the same parity with nearly degenerate excitation energies, transition probabilities and other characteristics [1]. Their inter-band $\mathrm{B}(\mathrm{E} 2)$ transition probabilities should be strongly suppressed in comparison with the intra-band $\mathrm{B}(\mathrm{E} 2)$ transitions rates [12]. A lot of partner bands were reported as candidates for chiral symmetry in different mass regions $\mathrm{A}=80$ [13], 100 [14-21], 130 [3$5,7,10,22-34]$ and $190[35,36]$. An odd A nucleus of ${ }^{135} \mathrm{Nd}[29]$ represent the chiral systems associated with a 3-quasiparticle configuration.

\footnotetext{
* Corresponding author: yavahchova@inrne.bas.bg
} 


\section{Nuclear dynamic chirality}

For the chiral partner bands is derived a set of indicators owing to experimental systematics of the nuclear chiral properties and a set of criteria which is needed to be fulfilled.

To investigate the setting up of chirality in a certain nucleus, it is important to determine the B(E2) and $\mathrm{B}(\mathrm{M} 1)$ values. In Ref. [37], it is demonstrated that the $\mathrm{B}(\mathrm{E} 2)$ and $\mathrm{B}(\mathrm{M} 1)$ pattern are not a unique fingerprint of chirality, some other characteristics are also important. Many lifetime measurements have been done, like for example for the nuclei of interest: ${ }^{134} \mathrm{Pr},{ }^{102} \mathrm{Rh},{ }^{135} \mathrm{Nd}$, ${ }^{194} \mathrm{Tl}$ and ${ }^{128} \mathrm{Cs}$. Lifetime results for ${ }^{134} \mathrm{Pr}$ are reported in the Refs. [7, 10]. For these nuclei the B(M1) values in both partner bands behave similarly. In contrast, the intraband $\mathrm{B}(\mathrm{E} 2)$ strengths within the two bands differ. In the spin region where the almost degeneracy of the energy levels of the two bands occurs, the B(E2) values for the yrast band are a factor 2 to 3 larger than those of the side band. The B(M1) staggering in both bands is not observed. In the case of ${ }^{128} \mathrm{Cs}$ the electromagnetic decay properties display the expected chiral pattern [31]. The structure in which a pair of twin bands is close in excitation energy, but the electromagnetic decay properties do not show the chiral pattern, in Ref. [37] is denoted as case A. The structure where the pair of twin bands is close in excitation energy and the electromagnetic decay properties display the chiral pattern, is denoted as case B. Odd-odd nuclei in the A $\sim 130$ mass region can be classified as case A or case B nuclei. In all these nuclei the cores are $\gamma$-soft, their oddproton odd-mass neighbours have also a similar structure and their odd-neutron odd-mass neighbours have a similar structure, too. There is no evident reason for the different structure of these nuclei, some of them being chiral (case B) and some being not chiral (case A). Either the structure of all these nuclei is not chiral, or there is a mechanism that dynamically induces chirality, in such a way that in case B it is far more pronounced than in case A [37].

The structure typical for case A means the nuclei to have twin bands, correct signature, different $\mathrm{B}(\mathrm{E} 2)$ values in the two bands, absence of $\mathrm{B}(\mathrm{M} 1)$ staggering in both bands and a very weak $\mathrm{B}(\mathrm{M} 1)$ staggering for $\Delta I=1$ transitions from the side to the yrast band. This structure is attributed to a weak dynamic (fluctuation dominated) chirality in Ref. [7]. The structure where the pair of twin bands is close in excitation energy and the electromagnetic decay properties display the chiral pattern, is denoted as case B. For case A the level energies for the yrast and side bands are becoming closer with increasing spin and cross at high spins, while in case B they are equidistant from the spin region where chirality sets up.

The chiral phenomenon is present in odd-odd, odd-A and even-even nuclei. It has been experimentally evidenced in the mass regions $\mathrm{A}=80,100,130,180$, 200 [38].

\section{Similarities in different cases}

Three good examples to express chirality in nuclei in different mass regions are ${ }^{128} \mathrm{Cs},{ }^{135} \mathrm{Nd}$ and ${ }^{194} \mathrm{Tl}$. We will make comparison between these nuclei and the two cases which we have been investigated - the cases of ${ }^{102} \mathrm{Rh}$ and ${ }^{134} \mathrm{Pr}$. Doppler-Shift Attenuation Method measurements have been utilized in all the experiments. The lifetimes and branching ratios were used to calculate the $\mathrm{B}(\mathrm{E} 2)$ and $\mathrm{B}(\mathrm{M} 1)$ reduced transition probabilities. In order to determine B(M1) values pure M1 transitions were supposed.

The first proposed candidate to express chirality was the odd-odd nucleus of ${ }^{134} \mathrm{Pr}$ with two $\Delta I=1$ rotational bands. The linking transitions are determined to be either stretched E2 or nonstretched $\Delta I=1 \mathrm{M} 1 / \mathrm{E} 2$ from the DCO analysis. Based on the multipolarities of these transitions, spin and parity of the side band are assigned. These two $\Delta I=1$ bands are almost degenerate Ref. [39]. In the mass region A 130 are existing conditions for the angular momentum coupling, due to the location in transition region with nuclear deformation between spherical and axially deformed shape. The first pair of $\Delta I=1$ bands with near energy degeneracy in an odd-mass nucleus was identified in ${ }^{135} \mathrm{Nd}$ [32]. The data for ${ }^{135} \mathrm{Nd}$ were taken from [32]. For mass region A 190 we chose to study ${ }^{194} \mathrm{Tl}$ and the data were taken from Ref. [40]. For $\mathrm{A} \sim 130$ the nucleus of ${ }^{128} \mathrm{Cs}$ was investigated, we used the results reported in Ref. [31]. These nuclei are representing some fingerprints for existence of static chirality. Reduced transition probabilities - B(M1) and $\mathrm{B}(\mathrm{E} 2)$, were derived from the measured lifetimes and are shown in Fig. 1 and Fig. 2. For the case of ${ }^{134} \mathrm{Pr}$ it is seen that the B(M1) values in both partner bands behave similarly and point to relatively strong transition strengths. The intraband $\mathrm{B}(\mathrm{E} 2)$ strengths within the two bands differ. These results are incompatible with pure chiral picture (static chirality) where the intraband $\mathrm{B}(\mathrm{E} 2)$ transition strengths must be almost equal $[1,6]$. This fact shows that static chirality is not reached in nucleus of ${ }^{134} \mathrm{Pr}$ and the nucleus stays in a very soft vibrational regime [10]. Moreover, the behavior of the chiral candidate bands is like in the discussed in the previous section - case A. For the nucleus of ${ }^{102} \mathrm{Rh}$, due to the limited statistic, we have determined only $B(E 2)$ values in Band 1. These B(E2) values show a similar behavior like in the yrast band of ${ }^{134} \mathrm{Pr}$. An extension to higher spins for the second band will answer the question whether the twin bands cross in excitation energies of the states. The excitation energies are similar, like it is both cases $\mathrm{A}$ and $\mathrm{B}$. We need to note that two bands could be called degenerate at least when the energy difference for analogue states is no more than $500 \mathrm{keV}$ [41].

For the case of ${ }^{135} \mathrm{Nd}$ the $\mathrm{B}(\mathrm{E} 2)$ and $\mathrm{B}(\mathrm{M} 1)$ values for the intraband transitions of the two bands are essentially the same, pointing to their identical nature. In addition, the $B(M 1)$ values of the intraband transitions exhibit a characteristic staggering with increasing spin. A similar staggering, but opposite in phase, is observed in the $\mathrm{B}(\mathrm{M} 1)$ values of the interband transitions [32]. 
All measured values for the $\mathrm{B}(\mathrm{M} 1)$ and the $\mathrm{B}(\mathrm{E} 2)$ transition probabilities in Bands 1 and 4 of ${ }^{194} \mathrm{Tl}$ nuclei
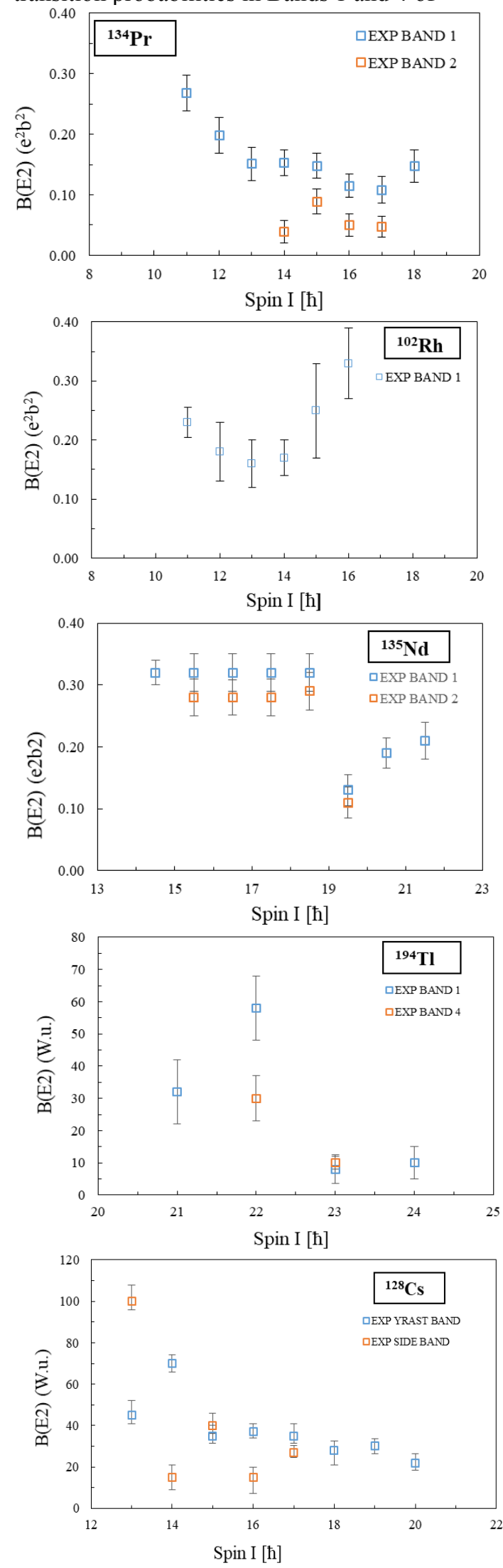

Figure 1. Experimentally determined B(E2) values for ${ }^{102} \mathrm{Rh}$, ${ }^{134} \mathrm{Pr},{ }^{135} \mathrm{Nd},{ }^{194} \mathrm{Tl}$ and ${ }^{128} \mathrm{Cs}$ are shown.
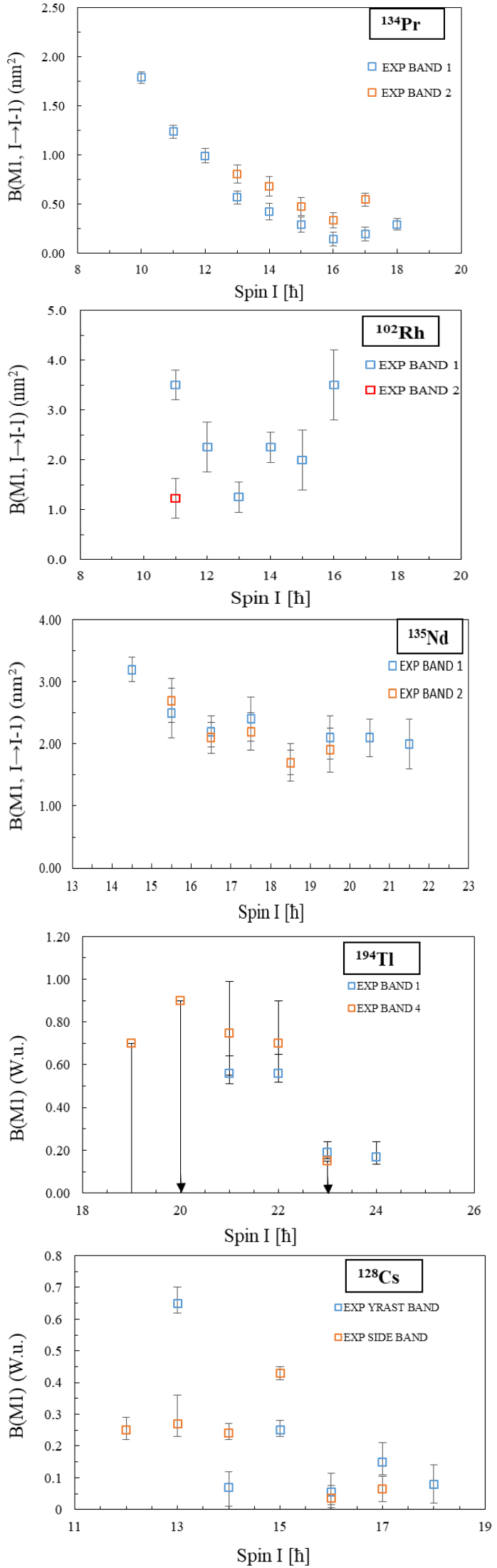

Figure 2. Experimentally determined B(M1) values for ${ }^{102} \mathrm{Rh}$, ${ }^{134} \mathrm{Pr},{ }^{135} \mathrm{Nd},{ }^{194} \mathrm{Tl}$ and ${ }^{128} \mathrm{Cs}$ are shown. 
look very similar. This strongly supports the suggested chiral symmetry of the two bands, and highlights the excellent near-degeneracy of this pair, making it perhaps one of the best chiral symmetry pair known until now [33].

The $\mathrm{B}(\mathrm{E} 2)$ and also $\mathrm{B}(\mathrm{M} 1)$ values are similar in the side and the yrast bands of ${ }^{128} \mathrm{Cs}$. The spin dependence of reduced M1 transition probabilities inside bands show characteristic staggering. The staggering of the B(M1) values is observed also for the side yrast transitions in ${ }^{128} \mathrm{Cs}[31]$.

For ${ }^{135} \mathrm{Nd},{ }^{194} \mathrm{Tl}$ and ${ }^{128} \mathrm{Cs}$ excitation energies are similar to case B and could be that conditions for static chirality are fulfilled.

The existence of staggering of B(M1) values is maybe the strongest test of static chirality. Figure 2 shows experimentally determined B(M1) values for all 5 nuclei of interest. It is obvious that in ${ }^{134} \mathrm{Pr}$ and in ${ }^{102} \mathrm{Rh}$ staggering is not seen. In the other 3 nuclei ${ }^{135} \mathrm{Nd},{ }^{194} \mathrm{Tl}$ and ${ }^{128} \mathrm{Cs}$ the existence of degenerate bands, the $\mathrm{B}(\mathrm{M} 1)$ and $\mathrm{B}(\mathrm{E} 2)$ similarity and an indication for staggering means that they fulfilled the requirement for existence of chirality. Also, their excitation energies are similar to the case $B$. The absence of the staggering of the $\mathrm{B}(\mathrm{M} 1)$ values in ${ }^{134} \mathrm{Pr}$ and in ${ }^{102} \mathrm{Rh}$, indicate that dynamic effects as coupling of the quasiparticles to fluctuations of the shape of the core may lead to differences in the properties of these bands [34]. Moreover, it is obvious that these nuclei revealing dynamic character of chirality. More experimental effort is needed in order to investigate the chirality in nuclei. An accelerator centre like we are building up in Sofia [42] will help the community to investigate the interesting phenomena like nuclear chirality.

\section{Summary}

Nuclear chirality is studied in five nuclei, namely ${ }^{134} \mathrm{Pr},{ }^{102} \mathrm{Rh},{ }^{135} \mathrm{Nd},{ }^{194} \mathrm{Tl}$ and ${ }^{128} \mathrm{Cs}$. The properties of the yrast and the side band are discussed. For nuclei ${ }^{134} \mathrm{Pr}$ and ${ }^{102} \mathrm{Rh}$ it is clearly seen that the requirement for existence of static chirality are not fulfilled. They support the presence of dynamic chirality of the type A [37]. In the cases of nuclei ${ }^{135} \mathrm{Nd},{ }^{194} \mathrm{Tl}$ and ${ }^{128} \mathrm{Cs}$, next to the existence of degenerate bands, B(M1) and $B(E 2)$ are similar and conditions for static chirality are fulfilled and they could be accepted as good examples for presence of nuclear chirality, type B [37].

The research has been supported by Bulgarian Science Fund under Contract No. 08/6, 13.12.2016.

\section{References}

[1] S. Frauendorf, Jie Meng, Nucl. Phys. A 617, 131 (1997)

[2] V. I. Dimitrov, S. Frauendorf, F Dönau, Phys. Rev. Lett. 84, 5732 (2000)
[3] K. Starosta, T. Koike, C.J. Chiara, D.B. Fossan, D.R. LaFosse, Nucl. Phys. A 682, 375 (2001)

[4] K. Starosta et al., Phys. Rev. C 65, 044328 (2002)

[5] T. Koike et al., Phys. Rev. C 67, 044319 (2003)

[6] T. Koike, K. Starosta, I. Hamamoto, Phys. Rev. Lett. 93, 172502 (2004)

[7] D. Tonev et al., Phys. Rev. C 76, 044313 (2007)

[8] S. Y. Wang, S. Q. Zhang, B. Qi, J. Meng, Chin. Phys. Lett. 24, 664 (2007)

[9] J. Meng and S. Q. Zhang, J. Phys. G: Nucl. Part. Phys. 37, 064025 (2010)

[10] D. Tonev et al., Phys. Rev. Lett. 96, 052501 (2006)

[11] D. Tonev et al., Phys. Rev. Lett. 112, 052501 (2014)

[12] S. Frauendorf, Rev. Mod. Phys. 73, 463 (2001)

[13] S.Y. Wang et al., Phys. Lett. B 703, 40 (2011)

[14] C. Vaman et al., Phys. Rev. Lett. 92, 032501 (2004)

[15] J. Timar et al., Phys. Lett. B 598, 178 (2004)

[16] J.A. Alcantara-Nunez et al., Phys. Rev. C 69, 024317 (2004)

[17] J. Joshi et al., Phys. Lett. B 595, 135 (2004)

[18] P. Joshi et al., Eur. Phys. J. A 24, 23 (2005)

[19] J. Timar et al., Phys. Rev. C 73, 011301(R) (2006)

[20] P. Joshi et al., Phys. Rev. Lett. 98, 102501 (2007)

[21] Y. X. Luo et al., Phys. Lett. B 670, 307 (2009)

[22] K. Starosta et al., Phys. Rev. Lett. 86, 971 (2001)

[23] R.A. Bark et al., Nucl. Phys. A 691, 577 (2001)

[24] A.A. Hecht et al., Phys. Rev. C 63, 051302(R) (2001)

[25] T. Koike et al., Phys. Rev. C 63, 061304(R) (2001)

[26] D.J. Hartley et al., Phys. Rev. C 64, 031304(R) (2001)

[27] G. Rainovski et al., Phys. Rev. C 68, 024318 (2003)

[28] A.A. Hecht et al., Phys. Rev. C 68, 054310 (2003)

[29] S. Zhu et al., Phys. Rev. Lett. 91, 132501 (2003)

[30] S. Wang et al., Phys. Rev. C 74, 017302 (2006)

[31] E. Grodner et al., Phys. Rev. Lett. 97, 172501 (2006)

[32] S. Mukhopadhyay et al., Phys. Rev. Lett. 99, 172501 (2007)

[33] E. Grodner et al., Phys. Lett. B 703, 46 (2011)

[34] A.D. Ayangeakaa et al., Phys. Rev. Lett. 110, 172504 (2013)

[35] E.A. Lawrie et al., Phys. Rev. C 78, 021305(R) (2008)

[36] P.L. Masiteng et al., Phys. Lett. B 719, 83 (2013)

[37] S. Brant et al., Phys. Rev. C 78, 034301 (2008)

[38] A.A. Raduta, Progress in Particle and Nuclear Physics 90, 241 (2016) 
[39] K. Starosta and T. Koike, Phys. Scr. 92, 093002 (2017)

[40] P.L. Masitheng et al., Eur. Phys. J. A 52, 28 (2016)

[41] M. S. Yavahchova et al., J. Phys.: Conf. Ser. 1023, 012020 (2018)

[42] D. Tonev, N. Goutev, L.S. Georgiev, J. Phys.:Conf. Ser. 724, 012049 (2016) 